\title{
Н.Л. Шураков
}

\section{ЯВЛЯЕТСЯ ЛИ 'ВОЛЕВОЙ АКТ' ПОНЯТИЕМ ЕСТЕСТВЕННОГО ВИДА? ${ }^{1}$}

\begin{abstract}
Рассматриваются различные трактовки понятий естественного вида в современной философии, а также влиятельные подходы к исследованию проблемы свободы воли, такие как компатибилизм, либертарианство и жесткий инкомпатибилизм. Приводится критика аргументов, позволяющих считать 'волевой акт' понятием естественного вида. Отстаивается позиция, что 'волевой акт' не является понятием естественного вида.

Ключевые слова: волевой акт, понятие естественного вида, компатибилизм, инкомпатибилизм.
\end{abstract}

Под ‘волевым актом’ в статье имеется в виду действие агента, в котором проявляется свобода воли. Ключевой характеристикой волевого акта является то, что он свободен. В рамках настоящей статьи такие понятия, как 'свободное действие / акт', 'акт свободы воли', 'волевое действие', используются как синонимы данного понятия. Вопрос «Является ли 'волевой акт' понятием естественного вида?» основан на разделении понятий на термины естественного (natural kind terms) и неестественного вида (non-natural kind terms). Такая дихотомия определяет, какие методы следует использовать, исследуя тот или иной вид. Например, классическим примером понятия естественного вида является 'вода'. Вода имеет химическую структуру $\mathrm{H}_{2} \mathrm{O}$ независимо от всех наших представлений о воде. Используя термин 'вода' люди в разное время ссылаются на определенное вещество, даже если они не знают ничего о его химической структуре. Вопрос «Что такое вода?» является скорее вопросом химии и физики, чем философии. По аналогии, если мы выясним, что 'волевой акт' является понятием естественного вида, то наше понимание волевого акта окажется независимым от того, насколько это действие свободно и чем оно обусловлено. Ответ на заглавный вопрос крайне актуален, поскольку бурное развитие когнитивных исследований ведет к тому, что свобода воли и волевой акт переходят из области интересов философов в область исследования когнитивистов. Положительный ответ ведет к выводу: волевой акт может быть исследован только эмпирически. Такой ответ может стать основой для ограничения методов исследования волевых актов лишь экспериментальными методами нейроученых. Следовательно, философский подход к проблеме утрачивает свою значимость. Однако вывод статьи позволяет обосновать обратное, т.е. важность именно философского исследования актов свободы воли.

Для ответа на поставленный вопрос в статье совершаются следующие шаги. В первой части обозреваются различные подходы к осмыслению понятий естественного вида, а именно: эссенциализм, кластерный подход и раз-

\footnotetext{
${ }^{1}$ Исследование выполнено за счет гранта Российского научного фонда, проект № 18-18-00222.
} 
нородный реализм. Во второй части приводятся основные взгляды на то, что же является 'волевым актом' согласно различным позициям в современной аналитической философии. Затем дается обзор некоторых аргументов в пользу того, что 'волевой акт' может рассматриваться как понятие естественного вида. В заключительной части статьи приводится аргумент Р. До и Т. Альтера против эссенциализма и кластерного подхода, а также указываются недостатки разнородного реализма Дж. Дюпре.

1. Повышенный интерес к понятиям естественного вида в XX в. отчасти связан с работами Н. Гудмана и У. Куайна, отчасти с Х. Патнэмом [1] и С. Крипке [2]. Последние нас интересуют больше, поскольку они предложили подход к понятиям естественного вида, получивший название эссенциализма. Эссенциалисты считают, что понятия естественного вида обозначают группы сущностей (essence). Сущности обладают определенными качествами и / или структурой, которые разделяются всеми членами естественного вида и присущи только членам этого вида. Например, определенная структура молекул углерода уникально обозначает термин естественного вида 'алмаз'. Все алмазы обладают одинаковой структурой и имеют одинаковую химическую формулу. Структура и формула алмаза определяют иные его свойства, такие как твердость, прозрачность и т.д. Важным качеством естественных видов является их определенность. Эссенциалисты считают, что должен существовать четкий критерий, позволяющий определить, к какому естественному виду относится тот или иной элемент. Не должно быть плавного перехода от одного естественного вида к другому, поскольку это сделало бы разграничение естественных видов произвольным. Так, Б. Эллис [3] считает, что определенность химических элементов является научным свидетельством, подтверждающим эссенциалисткую точку зрения. Важным свойством понятий естественного вида является наличие определенного фиксированного референта. Так, референт термина 'вода' и латинского 'аquа' одинаков. И тогда, и сейчас правильность употребления данных понятий связана с особой химической структурой $\mathrm{H}_{2} \mathrm{O}$. Римляне, даже не зная химической формулы воды, ссылались на то же вещество, на которое мы ссылаемся сейчас. Подчеркивая фиксированность референта понятий естественного вида, Крипке даже утверждает, что они являются жесткими десигнаторами, т.е. обозначают один и тот же объект во всех возможных мирах и не обозначают ничего иного. Однако эссенциалисткий подход малопригоден для схватывания естественных видов, обозначаемых различными биологическими классификациями. Сложно найти химическую структуру, присущую всем членам естественного вида, например кошкам. Как отмечает Э. Собер [4], практически невозможно определить свойства, принадлежащие всем членам определенного биологического вида и не принадлежащие иным видам. В частности, некоторые кошки могут являться носителями мутаций, при этом все равно оставаясь кошками.

Возможным решением эссенциалисткой проблемы выступает объединительный (unificationism), или кластерный, подход к понятиям естественного вида. Для образования естественного вида членам этого вида достаточно иметь некоторый общий набор свойств. Такие группы свойств образуют кластеры, например Felis silvestris catus объединяет всех домашних кошек и является понятием естественного вида. Биологи не идентифицируют виды, выделяя определенные сущностные качества, а исследуют филогенетическую 
связь организма с его предками, экологическую нишу и т.д. Организмы со сходными характеристиками объединяются в один биологический вид. По мнению одного из сторонников кластерного подхода Р. Бойда [5], биологические виды также являются и естественными, а понятия, их обозначающие, являются понятиями естественного вида.

Очевидная проблема объединительного подхода - отсутствие четкого определения кластерных свойств. Необходимо определить, какой набор свойств достаточен для их объединения в кластер. В то же время для одного естественного вида количество общих свойств может быть одним, а для иного другим. Четкое определение количества свойств может позволить объединить в естественный вид разнородные объекты и упустить некоторые в самом деле естественные виды. Таким образом, объединительный подход обременен проблемой разграничения терминов естественного вида от неестественного.

Представленные выше концепции Р. Уилсон объединил как позиции в рамках традиционного научного реализма [6. С. 306], противопоставляя этому взгляду разнородный (promiscuous) реализм Дюпре. Дюпре считает, что среди различных объектов встречается множество сходных свойств, на основании которых мы можем выделять естественные виды в зависимости от наших интересов и целей [7. С. 69]. Существует множество легитимных способов классификации объектов в мире. Разнородный реализм отрицает эссенциализм, считая что нет критерия для определения объекта в качестве члена естественного вида. Также отрицается и объединительный подход, формирующий иерархичные структуры. Естественные виды могут быть упорядочены различными способами. Дюпре считает, что классификации, присущие народной психологии, тоже имеют право на существование. Он приводит пример 'лилий' как понятия естественного вида, которое в рамках народной психологи объединяет биологически различные виды цветов. Классификации, распространенные в обыденном языке, могут не изменяться под влиянием научных, если в их основе лежит хотя бы одно общее свойство. Нам лучше принимать во внимание классификацию, более подходящую под конкретную цель. Преимуществом разнородного реализма является возможность классификации психологических расстройств в естественные виды. Когда мы имеем дело с психическими заболеваниями, нам довольно сложно выделить сущность заболевания на уровне химических элементов или определить характерные свойства, присущие конкретному заболеванию. Широта разнородного реализма порождает и его главный минус. Любые классификации, основанные на некоторой общности, могут трактоваться как естественные. Например, группа тревожных людей невероятно разнородна и, вероятно, только чувство тревоги является общим для всех членов этой группы.

Подводя предварительные итоги, заметим, что эссенциалистская трактовка понятий естественного вида наиболее узкая. Количество терминов естественного вида различно в зависимости от того, что имеется в виду под «понятием естественного вида». Однако легко заметить, что все эссенциалистские понятия естественного вида также подойдут и для кластерного подхода, и для разнородного реализма, а понятия кластерного подхода также сохранятся и в разнородном реализме.

2. Среди основных направлений исследований проблем, связанных со свободой воли, в аналитической философии мы рассмотрим компатибилизм 
(на примере взглядов Д. Деннета) и два варианта инкомпатибилизма: либертарианство Р. Кейна и жесткий инкомпатибилизм Д. Перебума. Для каждого подхода определим, чем же является 'волевой акт'.

Начнем с компатибилизма. Д. Деннет [8] придерживается компатибилисткой позиции, согласно которой в актуальном мире присутствуют и свобода воли, и детерминизм. Существование детерминизма в актуальном мире не ограничивает нашу способность совершать волевые акты и нести за них ответственность. Свобода воли, скорее, рассматривается Деннетом как характеристика личности, которая может помочь избежать нежелательных для организма последствий. Волевые акты сосуществуют с каузальными цепями и необходимы организму для его сохранения в мире.

Совместимость свободы воли с детерминизмом отрицается инкомпатибилизмом, одним из варинтов которого является либертарианство. Р. Кейн во многом возродил философский интерес к этой позиции [9]. Он считает, что для свободного выбора необходима ситуация «расколотой воли» [10. С. 167]. В такой ситуации у волящего агента имеется несколько возможных моделей поведения, для итогового выбора и поступка агент и совершает волевой акт. Кейн даже находит обоснование, как различные воли могут существовать на уровне физиологии: в этой ситуации активизируются разные группы нейронов. Позиция Кейна позволяет связать либертарианство и натуралистическую картину мира [11. С. 32]. Таким образом, волевым актом в либертарианстве можно считать итоговый поступок после ситуации «расколотой воли».

Наконец, в качестве представителя жесткого инкомпатибилизма расмотрим подход Д. Перебума. Он не принимает ни компатибилисткую, ни либертарианскую позицию, для него свобода воли одинаково несовместима ни с детерминизмом, ни с индетерминизмом. Согласно Перебуму, мы вообще не обладаем свободой воли, достаточной для моральной ответственности. Однако отказ от традиционных представлений о наличии свободы воли и моральной ответственности не влечет разрушения морали или утраты осмысленности жизни. Тем не менее, согласно такой позиции, мы вообще не совершаем волевых актов.

Соотнесем полученные трактовки 'волевого акта' с тремя подходами к понятиям естественного вида. Несложно заметить, что все трактовки предполагают реализм в отношении понятий естественного вида, поэтому сторонник жесткого инкомпатибилизма, отрицающий возможность поступать свободно, скажет, что 'волевой акт' не является понятием естественного вида. Поскольку актуальный мир определяется компатибилистами как мир, в котором сосуществуют и свобода воли, и детерминизм, а либертарианцами - как мир со свободой воли и индетерминизмом, то 'волевой акт' может оказаться понятием естественного вида. В качестве аргументов в пользу этого утверждения приведем аргументы А. Флю и М. Хеллера.

3. Флю разделяет атомарные и молекулярные выражения [12. С. 150151]. Молекулярные выражения мы узнаем исходя из определения, атомарные - из прямого указания на референт выражения. Как было обозначено выше, характеристикой 'волевого акта' является то, что он свободный. Флю считает, что быть свободным не является молекулярным выражением, поскольку оно не состоит из атомарных. Если мы выясним, что парадигмальные случаи, в которых мы обозначали совершенное действие как 'волевой акт', 
окажутся детерминированы, то обозначенные ранее действия все равно будут определяться термином «свободные». Семантическое значение «быть свободным» определяется остенсивно, поэтому у нас нет основания считать, что никакое действие не является свободным. Примечательно, что аргумент Флю сохраняет свою силу, даже если мы найдем подтверждение либертарианской позиции. Мы совершаем волевые акты независимо от того, чем и как обусловлены наши действия.

Ученик ван Инвагена М. Хеллер представил несколько иной аргумент [13] на основе аргумента Патнема в пользу того, что объем понятия определяется не нашим концептом, а прадигмальными случаями. Патнем предложил мысленный эксперимент следующего вида [14]: мы используем понятие 'кошка' применительно к животным различных видов семейства кошачьих. Исследуя кошек, мы однажды обнаруживаем, что все кошки в нашем мире на самом деле роботы, удаленно контролируемые инопланетянами. Если мы считаем, что понятие 'кошка' определяется концептом, согласно которому кошки - это живые млекопитающие организмы, то выяснив, что кошки на самом деле роботы, нам придется заключить, что в мире нет кошек. Напротив, если начинать с исследования парадигмального случая, то мы должны изучить все о произвольной кошке, чтобы определить, какие качества для нее являются необходимыми. Если мы в ходе исследований выясним, что произвольная кошка - это высокотехнологичный робот (и это не случайное ее качество), то мы узнаем что-то новое о кошках. В итоге наш вывод будет в том, что понятие 'кошка' обозначает определенных роботов.

Хеллер предлагает компатибилистам рассматривать понятие свободы воли аналогично. Он связывает свободу воли с поступком, который агент при желании может совершить или не совершить [13. С. 334]. (По сути, это лишь еще одна формулировка принципа альтернативных возможностей.) Отталкиваясь от такого определения, мы должны исследовать парадигмальные случаи свободных действий, постепенно узнавая все больше и больше о свободе воли. Например, можно прийти к тому, что для 'волевого акта' необходим принцип ответственности в конечном счете. Наше понимание понятия 'волевого акта' определяется парадигмальными случаями. Принимая аргументы Флю или Хеллера, можно отстаивать позицию, что 'волевой акт' - это понятие естественного вида, которое обозначает определенные свободные действия независимо от нашего понимания. Однако против этих аргументов можно привести возражения ван Инвагена и соместное возражение До и Альтера.

Ван Инваген [15. С. 106-112] демонстрирует, что использование аргумента Флю ведет к абсурду. Он предлагает представить, что в мозг каждого человека при рождении марсианами устанавливается необнаружимый крохотный девайс, контролирующий наши действия. При этом визуально такой возможный мир идентичен актуальному миру, использование языка также аналогично. Тогда остенсивное определение действий, охарактеризованных понятием 'свободные', в одном мире правильно, а в другом нет. Если предположение ван Инвагена окажется истинным, то нам следует признать, что мы не совершаем свободных действий. В то время как согласно аргументу Флю ‘волевыми актами' мы бы стали обозначать действия, совершенные под воздействием установленного девайса. 
Против аргумента Хеллера убедительное возражение приводят До и Альтер [16. С. 349-353]. Допустим, когда-либо ученые откроют, что определенное состояние головного мозга соотносится со всеми парадигмальными случаями, на основе которых мы сформировали понятие 'волевой акт'. Тогда метафизически возможны существа, совершающие волевые акты, но не испытывающие обозначенного состояния. Для любого состояния может быть дан подобный аргумент, поэтому До и Альтер заключают, что волевые действия не имеют естественной сущности, как вода или кошка. Таким образом, очевидно, что 'волевой акт' не может являться понятием естественного вида в эссенциалистском и объединительном подходах. Более того, если принять тезис множественной реализуемости свободы воли, то можно заключить, что ментальные состояния не образуют естественных видов, а ментальные предикаты не являются понятиями естественного вида [16. С. 355]. Так До и Альтер кратко формулируют позицию К. МакГинна в работе «Ментальные состояния, естественные виды и психофизические законы».

4. Представленные возражения достаточно сильно поддерживают утверждение, что 'волевой акт' не может являться понятием естественного вида в эссенциалистском и объединительном подходах. Аргументы Флю и Хеллера в пользу того, что 'волевой акт’ является понятием естественного вида, т.е. совершенно независим от наших представлений о нем, могут быть отклонены при помощи возражений ван Инвагена и До и Альтера. При разнородном реализме трактовка 'волевого акта' как понятия естественного вида возможна, но ведет к существенным проблемам. Например, все 'волевые акты' могут быть также обозначены как 'проявления воли летающего макаронного монстра', и такое определение будет иметь равное право на существование, но не будет представлять особого теоретического интереса. Уравнивание понятий 'волевой акт' и 'проявление воли летающего макаронного монстра' крайне сомнительно, но именно к этому приводит разнородный реализм. Признаем, что интерпретация 'волевого акта' в качестве понятия естественного вида крайне слаба. Таким образом, нам следует заключить, что 'волевой акт' понятием естественного вида не является. Данный вывод ведет к тому, что чисто эмпирическое исследование волевых актов не сможет предоставить полный ответ на вопрос: «Что такое волевой акт?» В исследовании волевых актов необходим плюрализм методов: научных и философских.

\section{Лuтература}

1. Putnam H. The meaning of 'meaning' // Minnesota Studies in the Philosophy of Science. 1975. № 7. P. 131-193.

2. Kripke S. Naming and Necessity. 12th td. Cambridge, MA : Harvard University Press, 2001.

3. Ellis B. Essentialism and Natural Kinds // The Routledge Companion to Philosophy of Science / ed. by M. Curd, S. Psillos. Routledge, 1999. P. 139-149.

4. Sober E. Evolution, Population Thinking and Essentialism // Conceptual Issues in Evolutionary Biology / ed. by E. Sober. Cambridge, MA : MIT Press, 1994. P. 161-189.

5. Boyd R. What Realism Implies and What It Does Not // Dialectica. 1989. № 43 (1-2). P. 5-29.

6. Wilson R. Promiscuous Realism // British Journal for the Philosophy of Science. 1996. № 47. P. 303-316.

7. Dupre J. Natural Kinds and Biological Taxa // The Philosophical Review. 1981. № 90 (1). P. 303-316.

8. Dennett D. Freedom Evolves. London : Penguin, 2007.

9. Kane R. The significance of free will. New York : Oxford University Press, 1996. 
10. Кейн Р. Свобода воли: ускользающий идеал / пер. с англ. Н.С. Поповой-Никитюк ; под ред. М.А. Секацкой // Философия. Журнал Высшей школы экономики. 2017. Т. I, № 4. С. 141199.

11. Мишура А. Поле битвы: свобода воли // Логос. 2016. № 5 (114). С. 19-58.

12. Flew A. Divine Omnipotence and Human Freedom // New Essays in Philosophical Theology / ed. by A. Flew, A. McIntyre. London : SCM Press, 1955.

13. Heller M. The Mad Scientist Meets the Robot Cats: Compatibilism, Kinds, and Counterexamples // Philosophy and Phenomenological Research. 1996. № 56 (2). P. 333-337.

14. Putnam H. It Ain’t Necessarily So // The Journal of Philosophy. 1962. № 59 (22). P. 658671.

15. Van Inwagen P. An Essay on Free Will. Oxford : Oxford University Press, 1983.

16. Daw R., Alter T. Free Acts and Robot Cats // Philosophical Studies. 2001. № 102. P. 345357.

Nikolai L. Shurakov, Saint Petersburg State University (Saint Petersburg, Russian Federation); University of Tartu (Tartu, Estonia).

E-mail: shurakovnukolay@gmail.com

Vestnik Tomskogo gosudarstvennogo universiteta. Filosofiya. Sotsiologiya. Politologiya - Tomsk State University Journal of Philosophy, Sociology and Political Science. 2020. 53. pp. 43-50.

DOI: $10.17223 / 1998863 X / 53 / 5$

IS A 'FREE ACT' A NATURAL KIND TERM?

Keywords: free act; natural kind term; compatibilism; incompatibilism.

The article is an attempt to answer the question of whether a 'free act' is a natural kind term. The answer to this question is of theoretical interest. An affirmative answer to the question leads to the conclusion: a free act can be investigated only empirically. The first part of the article reviews various approaches to the understanding of the concepts of natural kind terms, namely: essentialism, unificationism and promiscuous realism. The second part presents the main views on what a 'free act' is according to different positions in the contemporary analytic philosophy of mind: compatibilism (free will is compatible with determinism), libertarianism (there is free will and the world is not determined) and hard incompatibilism (free will is compatible with neither determinism nor indeterminism). It turns out that in hard incompatibilism, free acts could not form a natural kind. However, there is such an opportunity in compatibilism and libertarianism. For example, we can use the arguments by Antony Flew and by Michał Heller. Both arguments are applicable to compatibilism and libertarianism. In the final part of the article, objections from Peter van Invagen and the argument by Russell Daw and Torin Alter are considered. Van Inwagen argues that Flew's position leads to absurd. There is a possible world in which Martians put a tiny device in people's brains and control all their actions. But this world is identical to the real one if we agree with Flew. Daw and Alter provide a strong objection to Heller. If we find out that 'free acts' are defined by some essence or cluster of properties, then there possibly are organisms that lack this essence or these properties but act freely. Therefore, essentialism and unificationism could not define a 'free act' as a natural kind term. The last approach of promiscuous realism has the problem of finding the difference between natural and non-natural kinds. So, it is not the best way to argue that a 'free act' is a natural kind term. Thus, the conclusion of the article that a 'free act' is not a natural kind term.

\section{References}

1. Putnam, H. (1975) The meaning of 'meaning'. Minnesota Studies in the Philosophy of Science. 7. pp. 131-193.

2. Kripke, S. (2001) Naming and Necessity. 12th ed. Cambridge, MA: Harvard University Press.

3. Ellis, B. (1999) Essentialism and Natural Kinds. In: Curd, M. \& Psillos, S. (eds) The Routledge Companion to Philosophy of Science. Routledge. pp. 139-149.

4. Sober, E. (1994) Evolution, Population Thinking and Essentialism. In: Sober, E. (ed.) Conceptual Issues in Evolutionary Biology. Cambridge, MA: MIT Press. pp. 161-189.

5. Boyd, R. (1989) What Realism Implies and What It Does Not. Dialectica. 43(1-2). pp. 5-29. DOI: $10.1111 / \mathrm{j} .1746-8361.1989 . t b 00928 . x$

6. Wilson, R. (1996) Promiscuous Realism. British Journal for the Philosophy of Science. 47. pp. 303-316. DOI: $10.1093 /$ bjps/47.2.303 
7. Dupre, J. (1981) Natural Kinds and Biological Taxa. The Philosophical Review. 90(1). pp. 6690. DOI: $10.2307 / 2184373$

8. Dennett, D. (2007) Freedom Evolves. London: Penguin.

9. Kane, R. (1996) The Significance of Free Will. New York: Oxford University Press.

10. Kane, R. (2017) Free Will: The Elusive Ideal. Translated from English by N. PopovaNikityuk. Filosofia. Zhurnal vishey shkoly ekonomiki - Philosophy. Journal of the Higher School of Economics. 4. pp. 141-189. (In Russian). DOI: 10.17323/2587-8719-2017-I-4-141-189

11. Mishura, A. (2016) Battlefield - free will. Logos - The Logos Journal. 5(114). pp. 19-58. (In Russian).

12. Flew, A. (1955) Divine Omnipotence and Human Freedom. In: Flew, A. \& McIntyre, A. (eds) New Essays in Philosophical Theology. London: SCM Press.

13. Heller, M. (1996) The Mad Scientist Meets the Robot Cats: Compatibilism, Kinds, and Counterexamples. Philosophy and Phenomenological Research. 56(2), pp. 333-337.

14. Putnam, H. (1962) It Ain't Necessarily So. The Journal of Philosophy. 59(22). pp. 658-671. 15. Van Inwagen, P. (1983) An Essay on Free Will. Oxford: Oxford University Press.

16. Daw, R. \& Alter, T. (2001) Free Acts and Robot Cats. Philosophical Studies. 102. pp. 345357. 